\title{
Marburg virus-like particles protect guinea pigs from lethal Marburg virus infection
}

\author{
Kelly L. Warfield ${ }^{\text {a }}$, Dana L. Swenson ${ }^{\text {a }}$, Diane L. Negley ${ }^{\mathrm{a}}$, \\ Alan L. Schmaljohn ${ }^{\text {a }}$, M. Javad Aman ${ }^{\text {b,*, Sina Bavari }}{ }^{\mathrm{a}, *}$ \\ ${ }^{a}$ United States Army Medical Research Institute of Infectious Diseases, 1425 Porter Street, Frederick, MD 21702-5011, USA \\ ${ }^{\mathrm{b}}$ Clinical Research Management, Frederick, MD 21702, USA
}

Received 4 December 2003; received in revised form 16 January 2004; accepted 20 January 2004

Available online 4 March 2004

\begin{abstract}
Ongoing outbreaks of filoviruses in Africa and concerns about their use in bioterrorism attacks have led to intense efforts to find safe and effective vaccines to prevent the high mortality associated with these viruses. We previously reported the generation of virus-like particles (VLPs) for the filoviruses, Marburg (MARV) and Ebola (EBOV) virus, and that vaccinating mice with Ebola VLPs (eVLPs) results in complete survival from a lethal EBOV challenge. The objective of this study was to determine the efficacy of Marburg VLPs (mVLPs) as a potential vaccine against lethal MARV infection in a guinea pig model. Guinea pigs vaccinated with mVLPs or inactivated MARV developed MARV-specific antibody titers, as tested by ELISA or plaque-reduction and neutralization assays and were completely protected from a MARV challenge over 2000 LD $_{50}$. While eVLP vaccination induced high EBOV-specific antibody responses, it did not cross-protect against MARV challenge in guinea pigs. Vaccination with mVLP or eVLP induced proliferative responses in vitro only upon re-exposure to the homologous antigen and this recall proliferative response was dependent on the presence of CD4 ${ }^{+} \mathrm{T}$ cells. Taken together with our previous work, these findings suggest that VLPs are a promising vaccine candidate for the deadly filovirus infections. (c) 2004 Elsevier Ltd. All rights reserved.
\end{abstract}

Keywords: Marburg virus; Virus-like particle; Immunity; Vaccine

\section{Introduction}

Marburg (MARV) and Ebola (EBOV) viruses, members of the family Filoviridae, cause an acute and rapidly progressive hemorrhagic fever with mortality rates up to $90 \%$ $[1,2]$. The first recognized filovirus outbreak occurred in Marburg, Germany in 1967 after the importation of infected monkeys from Uganda [3,4]. Few other natural outbreaks of MARV have been recognized, with the most recent occurring in the year 2000. Based on outbreaks, the primary route of transmission is thought to involve direct contact with an infected individual or monkey; however, filoviruses are also transmissible via aerosols [3-5]. Unfortunately, the natural reservoir is not known for MARV. Additionally, MARV ap-

\footnotetext{
* Corresponding authors. Tel.: +1-301-619-4246; fax: +1-301-619-2290.

E-mail addresses: kelly.warfield@det.amedd.army.mil (K.L. Warfield), dana.swenson@det.amedd.army.mil (D.L. Swenson), diane.negley@det.amedd.army.mil (D.L. Negley), alan.schmaljohn@det.amedd.army.mil (A.L. Schmaljohn), javad.aman@det.amedd.army.mil (M.J. Aman) sina.bavari@amedd.army.mil, (S. Bavari).
}

pears to be fairly stable, even under harsh environmental conditions, and can survive in dried human blood for several days [5,6]. In a purported violation of the Biological Weapons Convention, MARV was actively pursued for use as a biological weapon [7]. All these factors make MARV a global health concern and the development of a vaccine is of paramount interest.

Survival from filovirus infection appears to correlate with the rapid initiation of immune responses. Filoviruses readily infect and replicate in mononuclear phagocytic cells, including monocytes, macrophages, and dendritic cells (DC) [8-11]. Conflicting reports indicate that filovirus-infected monocytes and macrophages either become highly activated and release large amounts of inflammatory chemokines and cytokines $[8,12]$ or they are unresponsive to filovirus-infection $[9,10]$. In support of the lack of immune responses to filovirus infections, EBOV and MARV infection of DC were shown to cause anomalous maturation of the infected DC $[10,11]$. Consequently, filovirus infection of DC does not elicit cytokine and chemokine secretion or induce the cell-surface co-stimulatory and adhesion molecules that are important 
for inflammation, DC migration, and lymphocyte stimulation $[10,11,13]$. The outcome of poor DC responses to filovirus infection probably includes deficient adaptive immune responses by $\mathrm{B}$ and $\mathrm{T}$ lymphocytes resulting in uncontrolled viral spread and growth. In contrast, the early initiation of pro-inflammatory responses correlates with the survival of EBOV-infected humans [14-17]. These findings indicate that rapid induction of the appropriate immune responses in humans can result in survival from filovirus infection. Therefore, it is plausible that a vaccination strategy that can effectively mobilize different arms of the immune system will induce protective immunity against filoviruses.

While no filovirus vaccines are currently licensed for human use, several vaccine candidates have been successfully tested for their ability to protect against MARV challenge with varying degrees of success [18-21]. A live, attenuated MARV isolate that causes viremia, but not disease or death, protects against lethal MARV challenge in guinea pigs [20]. Vaccination with formalin-inactivated MARV in the absence of adjuvant results in poor protection $(40-50 \%)$ in both guinea pigs and nonhuman primates $[21,22]$. In the presence of adjuvant, MARV inactivated by irradiation protected guinea pigs against challenge with a guinea pig-adapted MARV [18]. However, these classical approaches to development of a filovirus vaccine are unlikely to be useful in humans due to intractable problems for vaccine manufacturing in biosafety level-4 conditions and safety concerns for application in humans due to the possibility of incomplete inactivation, immunocompromized vaccine recipients, or revertant viruses [20]. Other studies have validated alternative vaccine approaches including DNA, vector-based, and subunit vaccines to induce protective immunity against MARV infection [18-20]. Vaccinating guinea pigs or nonhuman primates with a baculovirus-produced, secreted form of MARV GP or a DNA vaccine encoding GP is only partially protective $[18,20,23]$. However, a prime-boost strategy with both the DNA vaccine and baculovirus-produced GP completely protected animals challenged subcutaneously or by aerosol with MARV [20]. Additionally, a Venezuelan equine encephalitis replication-deficient particle (VRP) vaccine encoding MARV-Musoke GP was completely protective against homologous challenge with MARV-Musoke in guinea pigs and nonhuman primates $[19,20]$. These successful demonstrations of immunity to filoviruses are important; however, much remains uncertain about several of the alternative vaccine strategies employed thus far, including acceptable vaccine doses and safety considerations. Additionally, the impact of prior immunity to the vaccine vector and the ability of these vaccine strategies to cross-protect against multiple strains of MARV are of concern. Therefore, despite previous successes, developing a safe and efficacious vaccine against MARV remains a challenge.

Virus-like particle (VLP) vaccines are being tested in pre-clinical and clinical trials to protect against a wide array of viral diseases. VLPs are morphologically similar to the live virus from which they are derived and have proved to be a highly immunogenic means of antigen delivery, even in the absence of adjuvants [24,25]. Human papillomavirus VLPs are the best studied and have been shown to not only trigger the induction of neutralizing antibodies but also to induce specific $\mathrm{CD}^{+}{ }^{+} \mathrm{T}$ lymphocytes (CTL) responses in preclinical studies in nonhuman primates and humans $[26,27]$. VLPs are capable of mobilizing different arms of the adaptive immune system, are safe and effective in clinical settings, and raise no pre-existing or vector immunity concerns associated with other vaccine strategies such as VRP or adenovirus-based vaccines. Recently, we generated enveloped Ebola and Marburg VLPs (eVLPs and mVLPs, respectively) by expressing the viral glycoprotein and the matrix protein VP40 in mammalian cells [28-30]. The eVLPs are completely efficacious in preventing lethal EBOV infection in mice [29]. While mVLPs represent a promising novel subunit vaccine candidate, there are substantial differences in amino acid composition between Marburg and Ebola. Therefore, mVLPs need to be tested for efficacy against deadly MARV infection and the objective of the current work was to determine the immunogenicity and protective efficacy of mVLPs in a MARV guinea pig model.

\section{Methods}

\subsection{Virus and cells}

MARV or EBOV were propagated and enumerated by plaque assay on Vero E6 cells. MARV-Musoke or EBOV-Zaire 1995 virus preparations were purified over a continuous sucrose gradient and inactivated (i) by irradiation with $1 \times 10^{7} \mathrm{rad}$, as previously described [18]. MARV-or EBOV-infected cells and guinea pigs were handled under maximum containment in a biosafety level (BSL)-4 laboratory at the United States Army Medical Research Institute of Infectious Diseases. Convalescent serum samples removed from the BSL-4 laboratory were gamma-irradiated with $2 \times 10^{6} \mathrm{rad}$ from a ${ }^{60} \mathrm{Co}$ source before analysis in BSL-2 or BSL-3 laboratories [19,28,31].

\subsection{VLPS}

VLPs were prepared essentially as previously described, with minor modifications [28-30]. To generate mVLPs or eVLPs, 293T cells were co-transfected with pWRG 7077 vectors encoding for MARV or EBOV VP40 and GP using Lipofectamine 2000 (Invitrogen, Carlsbad, CA). To purify the VLPs, the cell supernatants were cleared from cellular debris and subsequently pelleted at $9500 \times g$ for $4 \mathrm{~h}$ in a Sorvall GSA rotor. The crude VLP preparations were then separated on a 20-60\% continuous sucrose gradient centrifuged in a SW41 rotor at $38,000 \mathrm{rpm}$ for $18 \mathrm{~h}$ (Beckman-Coulter, Inc., Fullerton, CA). The VLPs were concentrated by a second centrifugation and resuspended in endotoxin-free phosphate-buffered saline (PBS). The 
gradient fractions containing the VLPs were determined by western blots and electron microscopy. The mVLPs routinely sedimented in $\sim 35-50 \%$ sucrose, while the eVLPs sedimented in $\sim 30-40 \%$ sucrose. Total protein concentrations of the VLP preparations were determined after lysis in TritonX 100 detergent using a detergent-compatible protein assay (BioRad, Hercules, CA). The endotoxin levels in all VLP preparations used in this study were $<0.03$ endotoxin units $\mu \mathrm{g}$ by the Limulus amebocyte lysate test (Biowhittaker, Walkersville, MD).

\subsection{Electron microscopy}

Sucrose gradient-purified MARV or VLPs were processed as previously described [28]. Briefly, the particles were applied to formvar- and carbon-precoated grids. The grids were then treated with $1 \%$ glutaraldehyde, rinsed in distilled water, negatively stained with uranyl acetate, and examined on a JEOL EX transmission electron microscope at $80 \mathrm{kV}$.

\subsection{Vaccinations and Marburg virus challenge}

Inbred strain 13 guinea pigs (USAMRIID, Frederick, MD) were randomized into groups and each guinea pig was identified using a radio-transponder microchip (BioMedic Data Systems, Inc., Seaford, DE) inserted underneath the skin. Guinea pigs were vaccinated intramuscularly with $50 \mu \mathrm{g}$ of mVLPs $(n=5)$, eVLPs $(n=5)$, or iMARV $(n=5)$ with $200 \mu$ l of RIBI monophosphoryl lipid + synthetic trehalose dicorynomycolate + cell wall skeleton emulsion (Corixa Corporation, Hamilton, MT) diluted in endotoxin-free PBS on days 0,21 , and 42 . Control guinea pigs were vaccinated with RIBI adjuvant in PBS alone $(n=6)$. Serum samples were obtained from each guinea pig immediately before each vaccination and immediately prior to challenge (days $0,21,42$, and 72). The guinea pigs were challenged subcutaneously 30 days after the third vaccination (day 72) with $\sim 1000$ plaque-forming units (pfu) or $\sim 2000 \mathrm{LD}_{50}$ of guinea pig-adapted MARV diluted in PBS [18]. After challenge, guinea pigs were observed at least twice daily for illness and death. Research was conducted in compliance with the Animal Welfare Act and other federal statutes and regulations relating to animals and experiments involving animals and adhered to principles stated in the Guide for the Care and Use of Laboratory Animals, National Research Council, 1996. The facility where this research was conducted is fully accredited by the Association for Assessment and Accreditation of Laboratory Animal Care International.

\subsection{Antibody titers}

Levels of MARV and EBOV-specific antibodies were determined, as previously described [18]. Briefly, the wells were coated with sucrose-purified inactivated MARV or EBOV virions. Serial dilutions of each serum sample were tested and the endpoint titers were determined as the inverse of the last dilution where the optical density of the sample was $\geq 0.2$ greater than control wells (irrelevant heterologous antigen or wells without antigen).

\subsection{Plaque reduction-neutralization assay}

To determine the levels of plaque-neutralizing antibodies, two-fold dilutions of guinea pig sera were incubated with $\sim 100$ pfu of MARV at $37^{\circ} \mathrm{C}$ for $1 \mathrm{~h}$ in the presence of $5 \%$ guinea pig serum (as a source of complement), and then added to confluent Vero E6 cells [19]. A standard plaque assay with Vero E6 cells was performed and percent of plaque reduction was calculated by comparing the number of pfu present in each sample to the pfu obtained with virus alone $[19,32]$.

\subsection{Proliferation assay}

Single-cell suspensions were generated from the spleens of individual guinea pigs in RPMI-1640 medium containing $10 \%$ fetal bovine serum, $2 \mathrm{mM}$ L-glutamine, $1 \mathrm{mM}$ HEPES, and $0.1 \mathrm{mM}$ nonessential amino acids. As indicated, splenocytes were depleted of $\mathrm{CD}^{+}{ }^{+}$or $\mathrm{CD} 8^{+}$cells by negative selection using mouse anti-guinea pig CD4 or CD8 (Research Diagnostics, Inc., Flanders, NJ) and anti-mouse IgG magnetic beads (Dynal Biotech, Inc., Lake Success, NY). The total splenocytes or splenocytes depleted of $\mathrm{CD} 4^{+}$or $\mathrm{CD}^{+}$ $T$ cells were plated in 96-well culture plates at 200,000 cells per well in complete RPMI alone or with $10 \mu \mathrm{g} / \mathrm{ml}$ of eVLP or mVLP, as indicated. On day $5,1 \mu \mathrm{Ci}$ of ${ }^{3} \mathrm{H}$-thymidine was added to each well and the amount of ${ }^{3} \mathrm{H}$ incorporation was determined.

\section{Results}

\subsection{VLP vaccination induces humoral responses in guinea pigs}

The mVLPs were produced in cells transfected with MARV GP and VP40. After a purification procedure similar to authentic MARV, the mVLPs demonstrated remarkably similar morphology to filovirus virions (Fig. 1). We found both the MARV particles (Fig. 1a) and mVLPs (Fig. 1b) displayed similar heterogeneity, with particles of different lengths and shapes. In general, MARV appeared to be electron dense inside the viral particles, most likely due to the presence of the nucleocapsid proteins and RNA (Fig. 1a). However, some MARV particles appeared hollow, similar to the mVLPs, which contained only the glycoprotein and matrix proteins of MARV. Because the mVLPs and MARV had a similar morphology, but lacked potential virulence factors such as VP35 [10], we hypothesized that the genome-free mVLPs would be antigenically similar to MARV and, therefore, be useful as a vaccine against lethal MARV infection. 


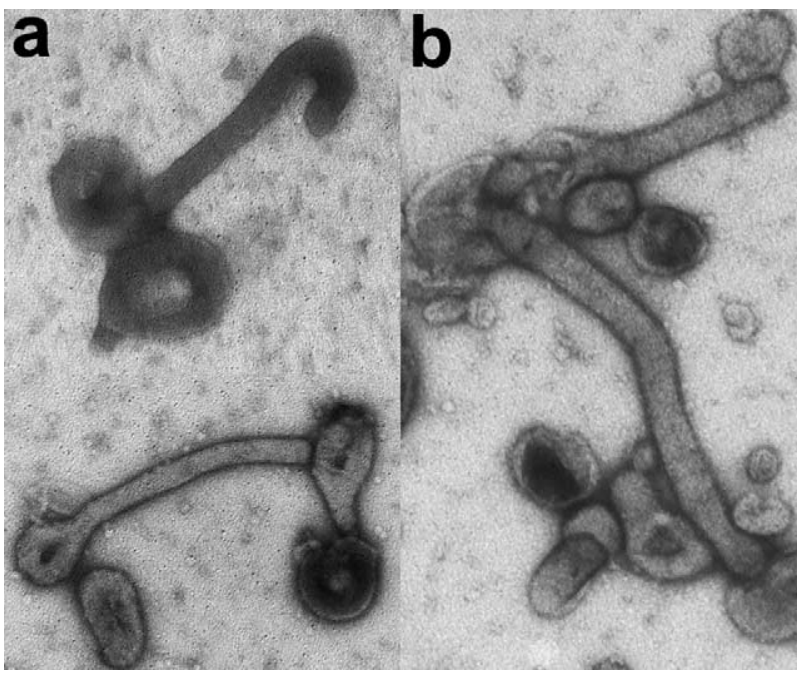

Fig. 1. Marburg virus-like particles (mVLP) are morphologically similar to authentic Marburg virus (MARV) virions. Electron micrographs of MARV (a) or mVLP (b) at 40,000 $\times$. Particles, obtained by ultracentrifugation of the supernatants of MARV GP and VP40 transfected cells or cells infected with MARV virus, were negatively stained with uranyl acetate to reveal the ultrastructure.

In guinea pigs, strong filovirus-specific antibody responses correlate with vaccine protective efficacy $[19,20,33]$. To assess the immunogenicity of the VLP vaccinations, groups of guinea pigs were vaccinated three times with inactivated MARV, mVLP, eVLP, or diluent and RIBI adjuvant. The guinea pigs were bled 21 days after each vaccination and the levels of MARV- or EBOV-specific antibodies were measured by ELISA (Fig. 2). mVLPs or inactivated MARV quickly elicited serum antibody responses to MARV after a single vaccine (Fig. 2a). Guinea pigs vaccinated three times with inactivated MARV developed MARV-specific antibodies in the range of 331,000-3,310,000. Similarly, guinea pigs vaccinated with $\mathrm{mVLP}$ developed high ELISA antibody titers against MARV after three doses (range: 10,000-331,000). Both inactivated MARV and mVLP induced maximal humoral responses to MARV after only two vaccinations (Fig. 2a). Although vaccination with inactivated MARV or mVLPs induced high titers of MARV-specific antibodies, it induced lower levels of cross-reactive antibodies against EBOV (Fig. 2b; endpoint titers ranged from 33,100 to 100,000 and 100 to 331 for inactivated MARV and mVLP, respectively). Conversely, guinea pigs vaccinated with eVLP acquired high serum antibody titers against EBOV, ranging from 331,000 to $1,000,000$ after three vaccinations (Fig. 2b). However, all of the eVLP-vaccinated guinea pigs had barely detectable levels of anti-MARV antibodies with endpoint titers of 331 (Fig. 2a). Guinea pigs vaccinated with adjuvant alone did not develop MARV- or EBOV-specific antibodies (Fig. 2a and b).

To evaluate the generation of neutralizing antibodies in the sera of the vaccinated guinea pigs, we used the plaque reduction-neutralization test $\left(\mathrm{PRNT}_{80}\right)$. Guinea pigs vaccinated with mVLPs developed neutralizing antibodies with a

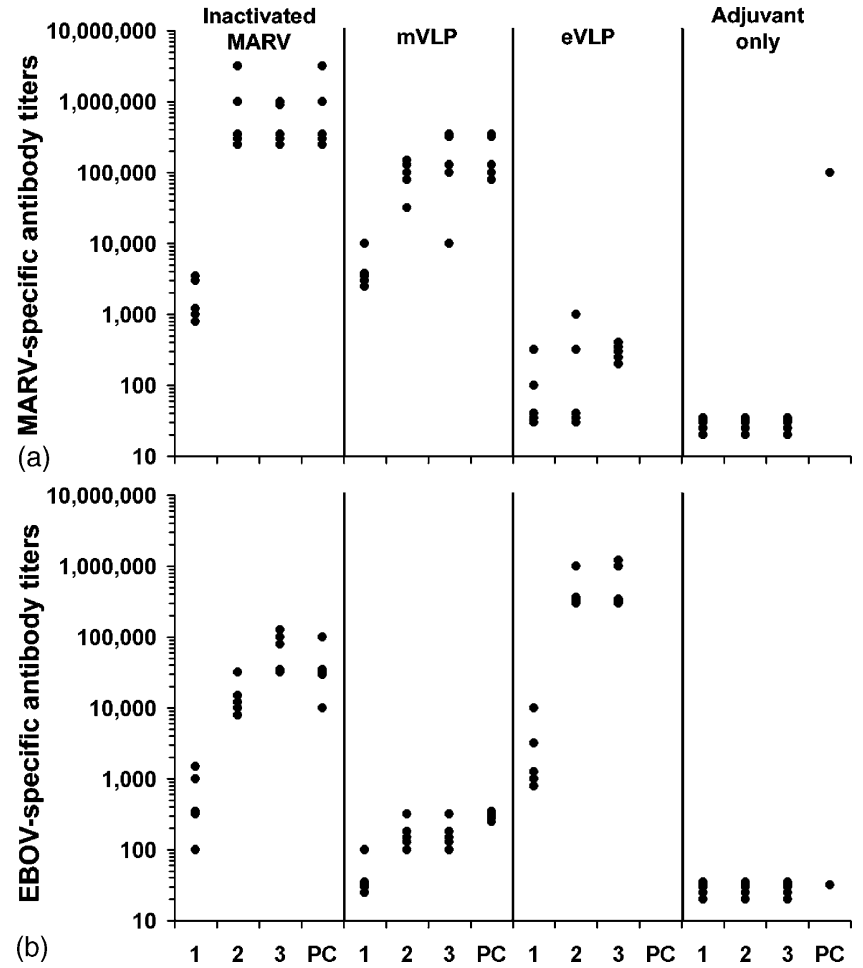

Fig. 2. Humoral responses to VLP vaccination. Strain 13 guinea pigs were vaccinated with iMARV $(n=5)$, mVLPs $(n=5)$, eVLPs $(n=5)$ in RIBI adjuvant, or adjuvant only $(n=6)$ three times at 3 -week intervals. ( $a$ and $b$ ) Serum samples from the guinea pigs were obtained 3 weeks after the first (1), second (2), or third (3) vaccination and 4 weeks after challenge (PC). Total serum (a) anti-MARV or (b) Ebola virus (EBOV) antibodies were measured by ELISA. Antibody titers were measured in serum from individual guinea pigs and the results are graphed as the individual endpoint titers for each guinea pig in each group.

PRNT $_{80}$ endpoint titer of 1:100 (Fig. 3, $n=5$ ). Guinea pigs that received inactivated MARV neutralized $80 \%$ or more of the virus up to a dilution of 1:300 $(n=5)$. However, guinea pigs that received eVLP or adjuvant alone were not able to significantly neutralize MARV infection of Vero E6 cells (Fig. 3). Considered together, these data indicate that mVLPs were able to induce high levels of MARV-specific antibodies, as well as neutralizing antibodies against MARV.

\subsection{VLP vaccination induces $C D 4^{+} T$ cell responses}

The generation of cellular immune responses is likely important for protection against pathogenic viruses, such as MARV and EBOV. Previously, Wilson et al. showed that cellular responses to EBOV NP are sufficient for protecting mice against lethal EBOV infection, demonstrating a critical role of T cells in filovirus immunity [34]. To assess the cellular immune responses generated after VLP injection, splenocytes from vaccinated guinea pigs were re-stimulated in vitro with mVLP or eVLP. Unfractionated T cells from guinea pigs vaccinated with eVLP or mVLP proliferated when re-exposed to the homologous, but not heterologous, antigen (Fig. 4a). To determine whether $\mathrm{CD}^{+}$or $\mathrm{CD} 8^{+}$ 


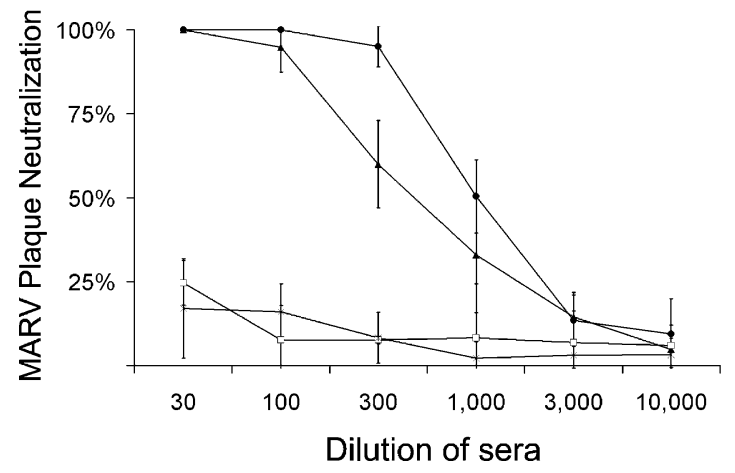

Fig. 3. Vaccination with mVLPs induces neutralizing antibody responses against MARV. Percent neutralization of MARV infection in Vero E6 cells by serum from guinea pigs vaccinated with inactivated MARV (iMARV) (filled circle, $n=5$ ), mVLP (filled triangle, $n=5$ ), or Ebola virus-like particles (eVLP, $n=5$ ) (open square) in RIBI adjuvant or adjuvant alone (star, $n=6$ ). Three-fold dilutions of serum were tested for their ability to neutralize MARV virus infection of VeroE6 cells and are plotted as the mean of the percent neutralization for each group of immune sera as compared to mock-treated Vero E6 cells. Error bars indicate the standard deviation of each group.

$\mathrm{T}$ cells were important for the recall memory responses to VLP vaccination, the splenocytes were depleted of CD4 ${ }^{+}$ or $\mathrm{CD}^{+} \mathrm{T}$ cells and the remaining cells were re-stimulated with VLPs. Depletion of $\mathrm{CD}^{+}$, but not $\mathrm{CD} 8^{+}$, $\mathrm{T}$ cells ablated the specific proliferative responses to VLP vaccination, indicating efficient priming of $\mathrm{CD} 4^{+} \mathrm{T}$ cells by VLP vaccination and suggesting a role for these cells in anti-MARV immune responses (Fig. 4b and c).

\section{3. mVLP vaccination induces protection against MARV challenge}

To determine whether mVLP vaccination could elicit protection from MARV challenge, groups of guinea pigs were vaccinated with three doses of inactivated MARV, mVLP, eVLP, or diluent and RIBI adjuvant and then challenged with 1000 pfu of guinea pig-adapted MARV-Musoke [18]. Guinea pigs vaccinated with mVLP or inactivated MARV were completely protected from lethal MARV infection (Fig. 5). Additionally, guinea pigs vaccinated with either mVLP or inactivated MARV did not show any visible signs of illness after MARV challenge (data not shown). In concert with lack of clinical symptoms after MARV challenge, the lack of increase in MARV-specific antibody levels after challenge (Fig. 2a) indicates that mVLP vaccination was able to effectively control MARV infection. In contrast, vaccination with eVLPs failed to protect animals from the related filovirus MARV (Fig. 5). eVLP-vaccinated guinea pigs succumbed to lethal MARV infection with kinetics very similar to guinea pigs vaccinated with adjuvant alone (Fig. 5). However, in the eVLP vaccines, MARV challenge appeared to initiate lethality earlier than the control guinea pigs. One guinea pig in the group of six vaccinated with RIBI adjuvant alone did not develop clinical signs

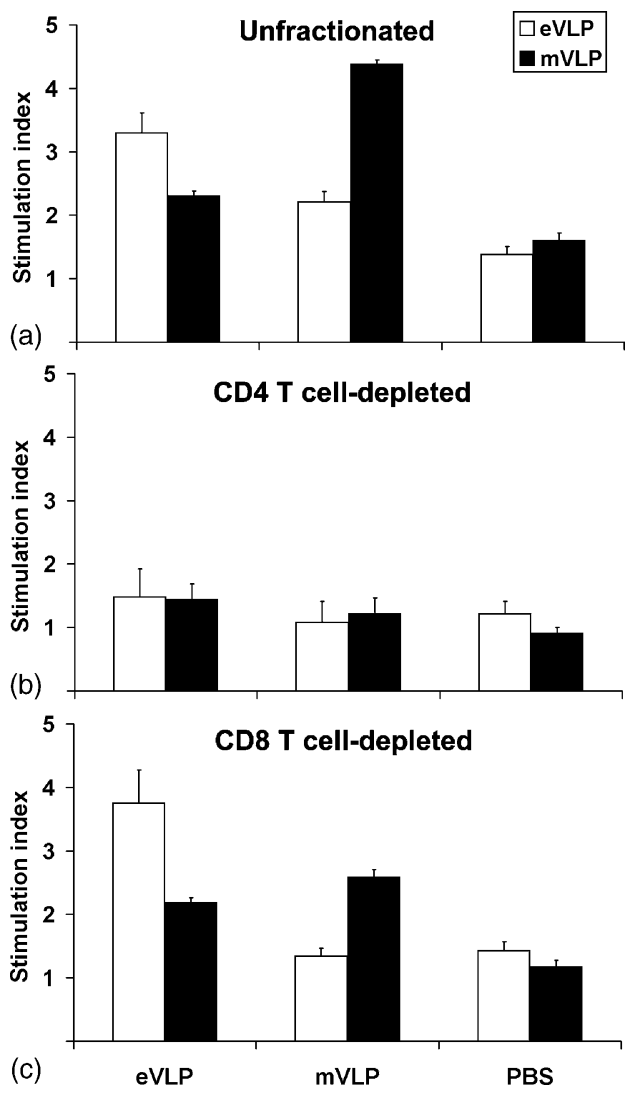

Fig. 4. VLPs induce recall $\mathrm{T}$ cell responses in guinea pigs. Unfractionated (a), $\mathrm{CD}^{+}$(b), or $\mathrm{CD}^{+}$(c) $\mathrm{T}$ cell-depleted splenocytes from guinea pigs vaccinated with mVLP, eVLP, or PBS in RIBI adjuvant were stimulated in vitro with mVLP, eVLP, or media alone for 6 days. During the last $18 \mathrm{~h}$ of culture, ${ }^{3} \mathrm{H}$-thymidine was added to each well and the amount of ${ }^{3} \mathrm{H}$ incorporation was assessed. The stimulation index was determined by dividing the ${ }^{3} \mathrm{H}$ incorporation in wells stimulated with eVLP (white) or mVLP (black) by the ${ }^{3} \mathrm{H}$ incorporation of wells cultured with media alone. The error bars represent the standard deviation of the mean of the stimulation index $(n=3)$.

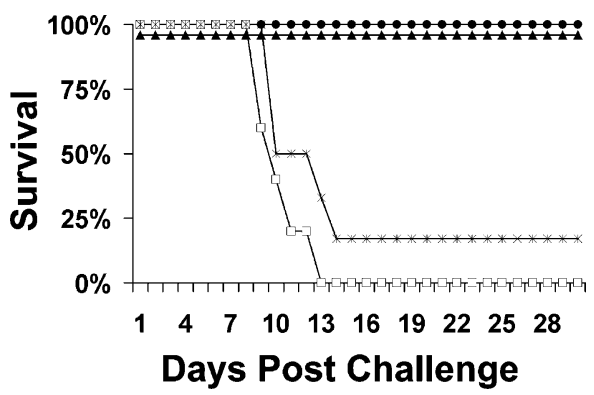

Fig. 5. Marburg VLPs protect guinea pigs against MARV challenge. Strain 13 guinea pigs were vaccinated with $50 \mu \mathrm{g}$ of inactivated MARV (iMARV) (filled circle), mVLP (filled triangle), or Ebola virus-like particles (eVLP) (open square) in RIBI adjuvant or adjuvant alone (star) three times at 3 -week intervals. All guinea pigs were challenged with 1000 pfu of guinea pig-adapted MARV-Musoke virus 5 weeks after the last vaccination. Results are plotted as percent survival for each vaccination group $(n=5-6$ per group). 
of filovirus infection and did not succumb to this lethal challenge dose of MARV (Fig. 5). After challenge with MARV, the lone survivor vaccinated with RIBI adjuvant displayed high MARV-specific antibody levels, indicating it was indeed exposed to MARV (Fig. 2a). Previous studies have shown that the guinea pig-adapted MARV-Musoke is not uniformly lethal, but causes death in 93\% (55/59) of Strain 13 guinea pigs $[18,19,28]$. Therefore, our results are in-line with previous data.

\section{Discussion}

In this study, we found that Marburg VLPs completely protected guinea pigs from lethal MARV. Vaccination with mVLPs induced strong humoral immune responses including high MARV-specific antibody titers and MARV plaque-neutralizing antibodies. Additionally, mVLP vaccination induced MARV-specific $\mathrm{CD}^{+}{ }^{+}$T-cell proliferative responses. Similarly, eVLPs induced high titers of EBOV-specific antibodies and T-cell proliferative responses in vaccinated guinea pigs. Not surprisingly, considering the limited amino acid homology $(\sim 31 \%)$ between EBOV and MARV, vaccination with eVLPs did not induce cross-reactive protection from MARV infection [2]. Although the efficacy of the eVLPs has not yet been tested against EBOV infection in guinea pigs, eVLP are highly efficacious in protecting against lethal challenge in a mouse model of EBOV infection [29]. Taken together, VLPs are promising vaccine candidates that circumvent the safety, production, or vector immunity concerns associated with other filovirus vaccine candidates.

VLP vaccination of guinea pigs induced high levels of total and neutralizing filovirus-specific serum antibodies. The role in protection of VLP-induced MARV-specific antibodies is unclear at this time, although serum from eVLP-vaccinated mice was insufficient to protect against lethal challenge in a mouse model of EBOV infection [29]. In contrast, passive transfer of antibodies from MARV-immune guinea pigs can protect naïve animals from MARV challenge in a dose-dependent manner [18]. Additionally, MARV-specific monoclonal antibodies can confer partial protection from MARV challenge in guinea pigs [35]. Together, these data indicate that a certain amount of antibodies with the appropriate specificity, isotype, and avidity are sufficient to protect against MARV infection in guinea pigs [18,36], as they are for EBOV infection in mice [32]. In this study we used RIBI adjuvant; however, we have previously shown that the mVLPs are immunogenic in mice in the absence of adjuvant and we are also testing the efficacy of the VLPs alone or in combination with other adjuvants, including QS-21 and mutant $E$. coli heat labile toxin LT(R192G) [30]. We were encouraged to find that vaccination with inactivated MARV or mVLP induced similar levels of MARV-specific total or plaque-neutralizing antibodies (Figs. 2 and 3). Additionally, vaccination with inactivated MARV or mVLP elicited levels of MARV-neutralizing antibodies similar to those previously reported after administration of filovirus vaccines or in convalescent animals [18-20,33,36]. Dissecting the role of antibodies in VLP-mediated protection is the focus of current studies in our laboratory.

The role of T-cell responses in protection against filovirus infection is also not well understood, but it is generally accepted that cellular immune responses are required to achieve complete protection against filovirus infection. Splenocytes from guinea pigs vaccinated with mVLPs specifically proliferated in culture in response to mVLP, but showed no proliferative response to eVLPs, while the opposite was true for guinea pigs vaccinated with eVLPs (Fig. 5). This proliferative response to VLPs required CD4 ${ }^{+}$ $\mathrm{T}$ cells, since depletion of $\mathrm{CD} 4^{+}$, but not $\mathrm{CD} 8^{+}$, cells ablated $\mathrm{T}$ cell stimulation. Similar to our findings, guinea pigs vaccinated with a prime-boost strategy of DNA and adenovirus vaccines encoding EBOV GP and NP, depletion of $\mathrm{CD} 4^{+}$, but not $\mathrm{CD} 8^{+}$, $\mathrm{T}$ cells reduced the recall responses to EBOV GP [37]. However, further functional assays with additional and appropriate antigens will be required to reach solid conclusions about the $\mathrm{CD}^{+}$and $\mathrm{CD}^{+}{ }^{+} \mathrm{T}$ cell responses in this model. While examining the role of specific cell types in guinea pigs in vivo is very difficult due to a lack of characterization and availability of antigens, depletion of cell types of interest, adoptive transfers, and knockout mice can be used to dissect the importance of specific immune components for protection against filovirus infection. Unfortunately, no mouse model is currently available for MARV. The mouse model of EBOV has been exploited to determine that successfully vaccinating mice with liposome-encapsulated irradiated $\mathrm{EBOV}$ requires $\mathrm{CD} 4^{+} \mathrm{T}$ cells. In contrast, using knockout mice, we found that $\mathrm{CD}^{+}$ $\mathrm{T}$ cells are required for eVLP-mediated protection from EBOV infection (Warfield, manuscript in preparation).

Cytotoxic T lymphocytes (CTLs) are proposed to be critical for protection against EBOV $[34,36]$. $\mathrm{CD}^{+} \mathrm{T}$ cells did not contribute to the recall response to VLPs in our culture system. It is well documented that memory $\mathrm{CD} 8^{+}$ $\mathrm{T}$ cells respond within hours of stimulation, as opposed to $\mathrm{CD}^{+}{ }^{+} \mathrm{T}$ cell recall responses, which can take days to regenerate [38]. Therefore, an inherent problem of antigen recall assays is their bias towards examining $\mathrm{CD}^{+}{ }^{+} \mathrm{T}$ cell responses and we think it is likely the timing of this particular assay may have masked any $\mathrm{CD} 8^{+} \mathrm{T}$ cell response toward the VLPs. Due to a lack of characterization of the guinea pig immune system, it is not currently possible to characterize the epitopes recognized by $\mathrm{CD} 8^{+} \mathrm{T}$ cells after VLP vaccination. For EBOV, several vaccine strategies including liposomes encapsulating inactivated EBOV, DNA prime/adenovirus boost, and alphavirus-replicon vaccines induce CTL responses against EBOV-specific epitopes of GP and/or NP in mice [31,33,34,39]. Evidence for the importance of these CTL responses was demonstrated when adoptive transfer of nucleoprotein-specific CTLs, but 
not antibody, conferred protection against lethal EBOV infection in naïve mice [34]. $\mathrm{CD}^{+}{ }^{+}$and $\mathrm{CD}^{+} \mathrm{T}$ cell responses are generated in mice vaccinated with eVLP [29]. For EBOV, there appears to be an absolute requirement for $\mathrm{CD}^{+} \mathrm{T}$ cells to achieve protection from lethal EBOV infection (Warfield, manuscript in preparation). While it is unclear at this time whether $\mathrm{CD}^{+}{ }^{+}$or $\mathrm{CD} 8^{+} \mathrm{T}$ cells are required for mVLP-induced immunity, it is likely that the generation of both effective $\mathrm{T}$ cell and humoral responses to filovirus antigens, especially glycoprotein, are critical.

This is the first report that eVLP-vaccination of guinea pigs efficiently induces humoral and cellular immune responses to EBOV and eVLP, respectively, and we are currently testing the efficacy of eVLP in guinea pigs. It is likely that eVLP will be efficacious against EBOV challenge in guinea pigs, based on our work that shows that eVLP are highly protective against EBOV infection in mice, even in the absence of adjuvant [29]. However, our current study shows that the cross-reactive immune responses induced by eVLP are not sufficient to protect against MARV infection. In fact, vaccination with eVLP tended to decrease the survival time following MARV challenge, when compared to control guinea pigs (Fig. 5). Other data indicate that in both rodents and nonhuman primates, ineffectual immune responses following vaccination with inactivated virus or other filovirus antigens can cause accelerated disease progression and an "early-death" phenomenon, when compared to naïve animals $([19,21,29,40]$ and data not shown). Several mechanisms could be responsible in an immune-mediated exacerbation of disease in unprotected animals, including mechanisms involving antibodies [41-43]. While the significance of this observation is not clear at this time, it could be important for consideration in future vaccine development and points to the importance of developing a pan-filovirus vaccine that broadly protects against all subtypes of both EBOV and MARV. To this end, VLPs provide an excellent system for generating broad-spectrum vaccines, since glycoprotein molecules from different filovirus strains can be efficiently incorporated into these particles (Swenson, manuscript in preparation). Future studies will focus on the evaluation of the efficacy of such hybrid vaccines, as well as mixtures of eVLP and mVLP, to protect against both EBOV and MARV.

In summary, we demonstrated that MARV and EBOV VLPs are highly immunogenic in guinea pigs, inducing both humoral and cellular responses against these filoviruses. Importantly, mVLPs completely protected animals against a high-dose parenteral MARV challenge. Marburg VLPs were highly efficacious with multiple advantages not offered by other candidate vaccines such as the safety of a subunit vaccine, no prior immunity to or interference by a vector, and presentation of the critical viral proteins glycoprotein and VP40 in a native form. This report extends our previous work, which demonstrated protective immunity in eVLP-vaccinated mice and provides further evidence to support future studies to evaluate the efficacy of VLPs for both MARV and EBOV in nonhuman primates. Considered together, our data indicate that VLPs have potential as a candidate vaccine for protection against filoviruses and may provide a tool to further dissect protective immune responses for MARV infection.

\section{Acknowledgements}

KLW and DLS are both the recipients of National Research Council Fellowships. We thank M. Gibson for excellent technical assistance, T. Larsen and K. Kuehl for help with electron microscopy, and C. Bosio, B. Moore, and M. Hevey for their support and insight. This work was sponsored by the Medical Research and Material Command Research Plan \#03-4-7J-021.

\section{References}

[1] Feldmann H, Klenk HD, Sanchez A. Molecular biology and evolution of filoviruses. Arch Virol Suppl 1993;7:81-100.

[2] Feldmann H, Klenk HD. Marburg and Ebola viruses. Adv Virus Res 1996;47:1-52.

[3] Martini GA, Seigert R, editor. Marburg virus disease. Berlin: Springer-Verlag; 1971.

[4] Smith DH, Johnson BK, Isaacson M, Swanapoel R, Johnson KM, Killey M, et al. Marburg-virus disease in Kenya. Lancet 1982;1(8276):816-20.

[5] Belanov EF, Muntianov VP, Kriuk VD, Sokolov AV, Bornotov NI, Plankov OV, et al. Survival of Marburg virus infectivity on contaminated surfaces and in aerosols. Vopr Virusol 1996;41:32-4.

[6] Frolov VGAG, M. Stability of Marburg virus to lyophilization process and subsequent storage at different temperatures. Vopr. Virusol. 1996;41:275-7.

[7] Alibek K, Handelsman S, Biohazard: random house. New York, NY, USA; 1999.

[8] Stroher U, West E, Bugany H, Klenk HD, Schnittler HJ, Feldmann $\mathrm{H}$. Infection and activation of monocytes by Marburg and Ebola viruses. J Virol 2001;75(22):11025-33.

[9] Gibb TR, Norwood Jr DA, Woollen N, Henchal EA. Viral replication and host gene expression in alveolar macrophages infected with Ebola virus (Zaire strain). Clin Diagn Lab Immunol 2002;9(1):19-27.

[10] Bosio CM, Aman MJ, Grogan C, Hogan R, Ruthel G, Negley $\mathrm{D}$, et al. Ebola and marburg viruses replicate in monocyte-derived dendritic cells without inducing the production of cytokines and full maturation. J Infect Dis 2003;188(11):1630-8.

[11] Mahanty S, Hutchinson K, Agarwal S, McRae M, Rollin PE, Pulendran B. Cutting edge: impairment of dendritic cells and adaptive immunity by Ebola and Lassa viruses. J Immunol 2003;170(6):2797801.

[12] Feldmann H, Bugany H, Mahner F, Klenk HD, Drenckhahn D, Schnittler HJ. Filovirus-induced endothelial leakage triggered by infected monocytes/macrophages. J Virol 1996;70(4):2208-14.

[13] Steinman RM. Dendritic cells and the control of immunity: enhancing the efficiency of antigen presentation. Mt Sinai J Med 2001;68(3):106-66.

[14] Baize S, Leroy EM, Georges-Courbot MC, Capron M, LansoudSoukate J, Debre P, et al. Defective humoral responses and extensive intravascular apoptosis are associated with fatal outcome in Ebola virus-infected patients. Nat Med 1999;5(4):423-6.

[15] Leroy EM, Baize S, Volchkov VE, Fisher-Hoch SP, Georges-Courbot MC, Lansoud-Soukate J, et al. Human asymptomatic Ebola infection and strong inflammatory response. Lancet 2000;355(9222):2210-5. 
[16] Leroy EM, Baize S, Debre P, Lansoud-Soukate J, Mavoungou E. Early immune responses accompanying human asymptomatic Ebola infections. Clin Exp Immunol 2001;124(3):453-60.

[17] Baize S, Leroy EM, Georges AJ, Georges-Courbot MC, Capron M, Bedjabaga I, et al. Inflammatory responses in Ebola virus-infected patients. Clin Exp Immunol 2002;128(1):163-8.

[18] Hevey M, Negley D, Geisbert J, Jahrling P, Schmaljohn A. Antigenicity and vaccine potential of Marburg virus glycoprotein expressed by baculovirus recombinants. Virology 1997;239(1):20616.

[19] Hevey M, Negley D, Pushko P, Smith J, Schmaljohn A. Marburg virus vaccines based upon alphavirus replicons protect guinea pigs and nonhuman primates. Virology 1998;251(1):28-37.

[20] Hevey M, Negley D, VanderZanden L, Tammariello RF, Geisbert J, Schmaljohn C, et al. Marburg virus vaccines: comparing classical and new approaches. Vaccine 2001;20(3-4):586-93.

[21] Ignatyev GM, Agafonov AP, Streltsova MA, Kashentseva EA. Inactivated Marburg virus elicits a nonprotective immune response in Rhesus monkeys. J Biotechnol 1996;44(1-3):111-8.

[22] Ignatev GM, Agafonov AP, Strel'tsova MA, Kuz'min VA, Mainagasheva GI, Spirin GV, et al. A comparative study of the immunological indices in guinea pigs administered an inactivated Marburg virus. Vopr Virusol 1991;36:421-3.

[23] Riemenschneider J, Garrison A, Geisbert J, Jahrling P, Hevey M, Negley D, et al. Comparison of individual and combination DNA vaccines for B anthracis, Ebola virus, Marburg virus and Venezuelan equine encephalitis virus. Vaccine 2003;21(25-26):4071-80.

[24] Johnson JE, Chiu W. Structures of virus and virus-like particles. Curr Opin Struct Biol 2000;10(2):229-35.

[25] Wagner R, Shao Y, Wolf H. Correlates of protection, antigen delivery and molecular epidemiology: basics for designing an HIV vaccine. Vaccine 1999;17(13-14):1706-10.

[26] Palker TJ, Monteiro JM, Martin MM, Kakareka C, Smith JF, Cook JC, et al. Antibody, cytokine and cytotoxic T lymphocyte responses in chimpanzees immunized with human papillomavirus virus-like particles. Vaccine 2001;19(27):3733-43.

[27] Harro CD, Pang YY, Roden RB, Hildesheim A, Wang Z, Reynolds MJ, et al. Safety and immunogenicity trial in adult volunteers of a human papillomavirus $16 \mathrm{~L} 1$ virus-like particle vaccine. J Natl Cancer Inst 2001;93(4):284-92.

[28] Bavari S, Bosio CM, Wiegand E, Ruthel G, Will AB, Geisbert TW, et al. Lipid raft microdomains: a gateway for compartmentalized trafficking of Ebola and Marburg viruses. J Exp Med 2002;195(5):593-602.

[29] Warfield KL, Bosio CM, Welcher BC, Deal EM, Mohamadzadeh M, Schmaljohn A, et al. Ebola virus-like particles protect from lethal
Ebola virus infection. Proc Natl Acad Sci USA 2003;100(26):15889_ 94.

[30] Swenson DL, Warfield KL, Kuehl K, Larsen T, Hevey MC, Schmaljohn A, et al. Generation of Marburg virus-like particles by co-expression of glycoprotein and matrix protein. FEMS Immunol Med Microbiol, 2004;40(1):27-31.

[31] Rao, M, Bray, M, Alving, CR, Jahrling, P, Matyas, GR. Induction of immune responses in mice and monkeys to Ebola virus after immunization with liposome-encapsulated irradiated Ebola virus: protection in mice requires $\mathrm{CD} 4(+) \mathrm{T}$ cells. $\mathrm{J}$ Virol 2002;76(18):9176-85.

[32] Wilson JA, Hevey M, Bakken R, Guest S, Bray M, Schmaljohn AL, et al. Epitopes involved in antibody-mediated protection from Ebola virus. Science 2000;287(5458):1664-6.

[33] Xu L, Sanchez A, Yang Z, Zaki SR, Nabel EG, Nichol ST, et al. Immunization for Ebola virus infection. Nat Med 1998;4(1):37-42.

[34] Wilson JA, Hart MK. Protection from Ebola virus mediated by cytotoxic T lymphocytes specific for the viral nucleoprotein. J Virol 2001;75(6):2660-4.

[35] Hevey M, Negley D, Schmaljohn A. Characterization of monoclonal antibodies to Marburg virus (strain Musoke) glycoprotein and identification of two protective epitopes. Virology 2003;314(1):350 7.

[36] Hart MK. Vaccine research efforts for filoviruses. Int J Parasitol 2003;33:583-95.

[37] Sullivan NJ, Sanchez A, Rollin PE, Yang ZY, Nabel GJ. Development of a preventive vaccine for Ebola virus infection in primates. Nature 2000;408(6812):605-9.

[38] Price DA, Klenerman P, Booth BL, Phillips RE, Sewell AK Cytotoxic $\mathrm{T}$ lymphocytes, chemokines and antiviral immunity. Immunol Today 1999;20(5):212-6.

[39] Vanderzanden L, Bray M, Fuller D, Roberts T, Custer D, Spik K, et al. DNA vaccines expressing either the GP or NP genes of Ebola virus protect mice from lethal challenge. Virology 1998;246(1):134 44.

[40] Ignatev GM. Immune response to filovirus infections. Curr Top Microbiol Immunol 1999;235:205-17.

[41] Takada A, Watanabe S, Okazaki K, Kida H, Kawaoka Y Infectivity-enhancing antibodies to Ebola virus glycoprotein. J Virol 2001;75(5):2324-30.

[42] Takada A, Feldmann H, Ksiazek TG, Kawaoka Y. Antibodydependent enhancement of Ebola virus infection. J Virol 2003;77(13):7539-44.

[43] Takada A, Kawaoka Y. Antibody-dependent enhancement of viral infection: molecular mechanisms and in vivo implications. Rev Med Virol 2003;13(6):387-98. 